m17.001

\section{Structure determination of nanosized, semi-coherent Al-Mg-Zn precipitates by precession electron diffraction and synchrotron X-ray diffraction}

Vidar Hansen $^{\mathrm{a}}$, Atle Kverneland ${ }^{\mathrm{a}}$, Gunnar Thorkildsen ${ }^{\mathrm{a}}$, Helge Bøvik Larsen ${ }^{\mathrm{a}}$, Philip Pattison ${ }^{\mathrm{b}}$, Cristopher Gilmore ${ }^{\mathrm{c}}$, Roger Vincent ${ }^{\mathrm{d}}$, Jon Gjønnes ${ }^{\mathrm{e}}$

${ }^{a}$ Faculty of Science and Technology, University of Stavanger, Norway. ${ }^{b}$ Swiss Norwegian Beam Lines, ESRF, Grenoble, France. ${ }^{c}$ Department of Chemistry, University of Glasgow, UK. ${ }^{d} H . H$. Wills Laboratory, University of Bristol, UK. ${ }^{e}$ Centre for Materials Science and Nano Technology, University of Oslo, Norway.

Keywords: structural analysis, precession electron diffraction, synchrotron X-ray diffraction

The $\eta$ '-precipitate is the main strengthening agent in age-hardening Al-Mg-Zn alloys. A new structure model of the phase has been derived by a combination of electron microscopy techniques and synchrotron X-ray diffraction. Samples for intensity measurements were prepared from an alloy casting with large matrix grains alloyed with $88.1 \mathrm{Al}, 10.2 \mathrm{Zn}$, and 1.68 $\mathrm{Mg}$. wt per cent, after aging by a standard procedure. Electron microscope images reveal a distribution of precipitates 3-10 nm wide, with varying degree of stacking disorder, in four different lattice orientations, embedded in the aluminium matrix. Electron diffraction patterns confirmed the hexagonal lattice, $\mathrm{a}=0.496$ $\mathrm{nm}, \mathrm{c}=1.405 \mathrm{~nm}$ reported earlier. Intensities were measured by the precession technique [1], which suppressed multiple scattering via matrix reflections, but incurred a high background of diffuse scattering. Systematic absences are consistent with three hexagonal, $\mathrm{P}_{3} \mathrm{mc}(186), \mathrm{P} 62 \mathrm{c}(190)$ and $\mathrm{P}_{3} / \mathrm{mmc}(194)$ or two trigonal space groups, P31c (159) and P31c (163). Due to the extensive overlap and high background level, we could not distinguish between a modified version in (190) of the earlier model [2] or a trigonal structure in (163) from electron diffraction data.

Three-dimensional synchrotron data were collected at the Swiss Norwegian Beamlines at ESRF from two single aluminium grains. More than 2000 intensities were extracted from each of the four orientations of these precipitate. The preliminary analysis points towards an average structure best described in the centrosymmetric space group P31c.

A trigonal model adopted for an average $\eta$ '-structure can be described as a faulted stacking of units of the Laves phase stable $\mathrm{MgZn}_{2}$, in a way that retains the trigonal stacking - as in the parent FCC aluminium lattice.

[1] R. Vincent and P.A. Midgley, Ultramicroscopy 1994, 53, 271

[2] X.Z. Li, V. Hansen, J. Gjønnes and L.R. Wallenberg, Acta Mater. 1999, 47, 2651.

\section{m17.002}

\section{How to resolve ab-initio nanostructures by electron diffraction: applications of beam precession in transmission electron microscopy}

\author{
$\underline{\text { Stavros Nicolopoulos }}^{\mathrm{a}, \mathrm{b}}$, Maximilian Nickolskiy ${ }^{\mathrm{b}, \mathrm{c}}$
}

${ }^{a}$ Universidad Politecnica de Valencia Avda de los Naranjos s/n 46022 Valencia Spain, ${ }^{b}$ NANOMEGAS Blvd Edmond Machtens 79 B-1080 Brussels, Belgium, ${ }^{c}$ Institute of Ore Mineralogy (IGEM) RAS Staromonetny per 35 Moscow 109017 Russia. E-mail: info@nanomegas.com

\section{Keywords: beam precession, electron diffraction, nanostructures}

To study crystal structure of individual nanocrystals electron microscopy is superior than single crystal ( or powder) X-Ray. In transmission electron microscopy (TEM) crystals of nm size can be examined; electron interaction with matter is about $10^{4}$ stronger than X-Rays and as a result strong dynamical scattering may alter diffracted intensities so much that cannot be trusted for crystal structure refinement, unless crystal thickness is very thin. This is why electron crystallography was not until now routine structure identification tool like X-Ray crystallography where beam-matter interactions are kinematical and structure factors can be directly derived from intensity data. Electron beam precession technique proposed by Vincent, Midgley [1], gives a solution to this problem by increasing the kinematical character of the electron diffraction. This technique is equivalent to the X-Ray Buerger precession technique, where instead precessing the crystal (like in X-Ray precession), electron beam is tilted and precessed on a cone surface having a common axis with the TEM optical axis. To obtain a stationary precession diffraction pattern instead of ring reflections (fig.1) a simultaneous descan of the diffracted beam is realized by means of image shift TEM coils. As a result of electron beam precession, only few reflections are simultaneously excited in dynamical conditions and the resulting diffraction pattern can be considered close to kinematical or two beam conditions.In the resulting diffraction pattern kinematically forbidden reflections and multiple scattering are virtually eliminated (or greatly reduced) and space group identification can be easily done.By using quasi-kinematical precession intensities, several structures could be solved ab-initio as minerals [2],catalysts [3], and complex oxide structures [4]. Is interesting to note that $3 \mathrm{~d}$ complex structures like mineral phlogopite mica can be resolved using only a few zone axis precession reflections, while all atoms (including light oxygens) can be identified precisely.

This work is supported by RFBR, Project 050565242 .
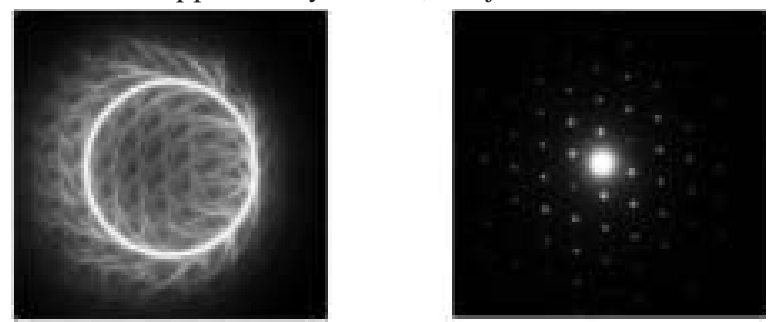

FIG. 1 (left) precession diffraction pattern of [110] Si without and with descan (right)

[1] Vincent R.,Midgley P., Ultramicroscopy, 1994, 271

[2] Gemmi M, Nicolopoulos S, Ultramicroscopy, special issue 2006 (to be published).

[3] Dorset D., Gilmore C.,Jorda JL.,Nicolopoulos S., Ultramicroscopy, special issue 2006 (to be published).

[4] Weirich T., Portilo J., Cox G., Nicolopoulos S., Ultramicroscopy, 106,164 . 